15

\title{
Микроволновая вольт-импедансная спектроскопия полупроводников
}

\author{
(c) А.Н. Резник, Н.В. Востоков, Н.К. Вдовичева, В.И. Шашкин \\ Институт фризики микроструктур РАН, \\ 603950 Нижний Новгород, Россия \\ e-mail: reznik@ipm.sci-nnov.ru
}

Поступило в Редакцию 3 апреля 2020 г.

В окончательной редакции 3 апреля 2020 г.

Принято к публикации 3 апреля 2020 г.

Предложенный ранее авторами метод микроволновой вольт-импедансной спектроскопии полупроводников апробирован в эксперименте. Метод позволяет определить локальные значения электрофизических параметров полупроводника. Исследования выполнены на однородной монокристаллической пластине $\mathrm{GaAs}$, поверх которой сформирована система концентрических антенн. Разрешающая способность определяется диаметром центрального диска антенны, который составлял 12, 27, $57 \mu \mathrm{m}$. Постоянное напряжение смещения $0 \leq U \leq 5 \mathrm{~V}$ прикладывалось между контактными площадками антенн. Спектр комплексного импеданса каждой антенны $Z(f, U)$ измерялся при помощи зондовой станции Cascade Microtech в диапазоне частот $f=0.1-10 \mathrm{GHz}$. Электрофизические характеристики полупроводника определялись по спектрам $Z(f, U)$ путем решения обратной задачи. Установлен $n$-тип полупроводника и определена контактная разность потенциалов на границе с металлом. Найдены локальные значения концентрации и подвижности электронов, удельной электропроводности. Измерения средних по поверхности значений этих же параметров холловским четырехзондовым методом показали хорошее взаимное соответствие результатов для исследованной однородной пластины.

Ключевые слова: ближнепольная микроскопия, антенна, микроволновый импеданс, частотный спектр, полупроводник.

DOI: 10.21883/JTF.2020.11.49988.115-20

\section{Введение}

Измерение вольт-фарадных характеристик - один из основных инструментов диагностики полупроводников $(C-V$-метод), позволяющий определить концентрацию свободных носителей заряда, а в более общем случае - глубинный профиль концентрации. Одновременное измерение емкости и проводимости дополнительно позволяет получить дрейфовую подвижность носителей [1-4]. До недавнего времени соответствующие измерения выполнялись на низких частотах (менее $10 \mathrm{MHz}$ ), вследствие чего электрофизические характеристики могли быть получены с латеральным разрешением не более $0.1 \mathrm{~mm}$. В последние годы в связи с развитием микроволновых методов, в частности, с созданием коммерческой зондовой системы Cascade Microtech (CM), открылась возможность локальных импедансных измерений в микроволновой части спектра [5-7]. Подобные измерения могут выполняться в условиях обеднения поверхностного слоя полупроводника за счет приложенного электрического поля $[5,7]$. Перспектива такой диагностики видится в реализации микронной и нанометровой латеральной разрешающей способности $C-V$-метода. Дело в том, что при диаметре емкостного контакта $<10 \mu \mathrm{m}$ чувствительность измерительного устройства в радиочастотном диапазоне оказывается недостаточной, так как измеряемая емкость $C<1 \mathrm{fF}$. С точки зрения чувствительности микровол- новые методы предпочтительнее, поскольку емкостной импеданс $Z$ уменьшается обратно пропорционально частоте $f(X=-\operatorname{Im} Z=1 /(2 \pi f C))$, что понижает предел измеримой емкости до требуемого уровня. Другое важное обстоятельство заключается в том, что измерение не только мнимой, но также и действительной части импеданса $R=\operatorname{Re} Z$ (т. е. $Z-V$-спектроскопия) позволяет получить полный набор электрофизических характеристик полупроводника: концентрацию $n_{0}$, подвижность $\mu$ и тип свободных носителей заряда, а также удельную проводимость $\sigma_{0}$. Теоретическое обоснование возможности соответствующей диагностики дано в работе [8].

В последние годы для локальной бесконтактной диагностики полупроводниковых материалов и структур применяется микроволновая микроскопия. Измерения выполняются в ближнем поле зонда (антенны), вследствие чего достигается субволновое пространственное разрешение, которое по порядку величины равно линейному размеру апертуры антенны. Микроскопия полупроводниковых структур в микроволновом диапазоне с латеральным разрешением от десятков нанометров до сотен микрометров производилась в работах [9-14], где изучалась возможность определения проводимости $\sigma$, либо концентрации $n_{0}$. Емкостная чувствительность современных микроволновых микроскопов достигает $1 \mathrm{aF}$ [10]. Если на зонд микроскопа дополнительно подать постоянное напряжение смещения $U$, создающее в окрестности ближнепольной антенны обедненный/обогащенный 
слой, толщина которого определяется невозмущенной концентрацией $n_{0}$, то появится возможность бесконтактно определить вышеперечисленные электрофизические параметры полупроводника. Исследования по $C-V$-диагностике полупроводников при помощи сканирующей микроволновой микроскопии нанометрового разрешения активно ведутся в последние годы [15-17]. Указанные работы ограничивались попытками определения концентрации $n_{0}$ и из-за ряда технических сложностей пока не достигли необходимой точности.

В настоящей работе выполнена экспериментальная реализация предложенного в [8] метода микроволновой $Z-V$-спектроскопии. Продемонстрирована возможность неразрушающего определения вышеперечисленных электрических характеристик полупроводника с микронным латеральным разрешением.

\section{1. Методика измерений и исследованный образец}

Исследовалась однородная в латеральной плоскости и по глубине подложка GaAs толщиной $0.43 \mathrm{~mm}$. На подложке была сформирована система концентрических барьерных контактов (антенная система), для чего на поверхность полупроводника последовательно наносились слои $\mathrm{Al}(50 \mathrm{~nm}), \mathrm{Ni}(20 \mathrm{~nm})$ и $\mathrm{Au}(80 \mathrm{~nm})$ в окнах фоторезистивной маски методом электроннолучевого испарения, с последующим удалением фоторезиста. Каждый $1.5 \times 1.5 \mathrm{~mm}$ пиксель антенной системы представлял собой решетку из девяти дисков, встроенных в металлический квадрат со стороной $c=0.5 \mathrm{~mm}$, как показано на рис. 1. Квадраты разделены между собой непроводящей щелью. Аналогичная конфигурация антенной системы использована для измерений в [5-7].

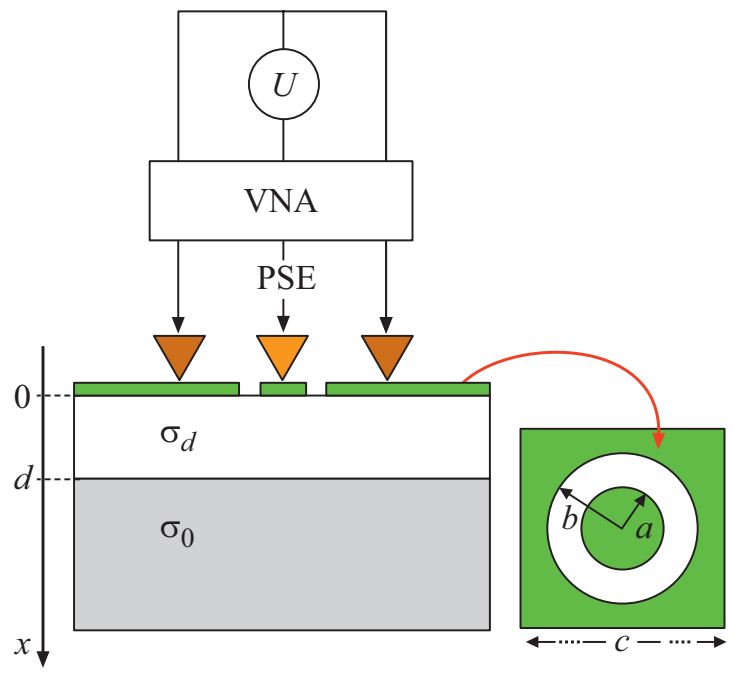

Рис. 1. Схема измерений импеданса зонда и полупроводниковая структура с контактными поверхностями антенны. VNA векторный анализатор цепей, PSE - электроды зондовой станции Cascade Microtech. На вставке справа - геометрия коаксиальной антенны.
В микроволновых измерениях мы использовали три из девяти антенн с радиусами $a, b=6,26.5 \mu \mathrm{m}$ (1); 13.5, $34 \mu \mathrm{m}(2) ; 28.5,49 \mu \mathrm{m}$ (3) соответственно.

Зонд Cascade Microtech в виде симметричной копланарной линии, приведенной в контакт с тестовой структурой, подключался к векторному анализатору цепей Agilent E8361 A (рис. 1). Постоянное напряжение $U$ подавалось на электроды СМ зонда от внешнего источника через развязанную с микроволновым трактом низкочастотную цепь, встроенную в анализатор цепей. Измерялся комплексный коэффициент отражения S11 зондирующего сигнала от структур в диапазоне частот $f=0.01-10 \mathrm{GHz}$. После калибровки измерительной системы с использованием стандартного набора калибровочных мер определялся микроволновый спектр комплексного импеданса антенны $Z(f)=R(f)-i X(f)$. Измерения спектра $Z(f)$ выполнялись при нескольких допробойных значениях напряжения в диапазоне $0 \leq U \leq 5 \mathrm{~V}$. Методика подобных измерений более детально описана в работах [5-7]. Как показано в работе [6], в указанном диапазоне значений $a, b$ радиус $b$ не влияет на импеданс антенны $Z$, а латеральная разрешающая способность системы определяется величиной диаметра центрального контакта $2 a$, т. е. в нашем случае реализовано разрешение $\sim 10-60 \mu \mathrm{m}$. Отметим, что использованная многопиксельная антенная система позволяет исследовать полупроводниковые структуры с неоднородным распределением электрических характеристик в плоскости поверхности образца [6]. Для исследуемой однородной подложки измерения выполнялись на одном пикселе в центральной части образца.

С изменением напряжения $U$ меняется толщина $d$ слоя возмущенной проводимости. В рассматриваемом диапазоне $U$ в слое $x<d$ имеет место обеднение концентрации основных носителей, т.е. эффективная проводимость полупроводника понижена $\sigma_{d} \ll \sigma_{0}$ (рис. 1 ). Вне слоя проводимость принимает невозмущенное значение $\sigma_{0}$. При $U=0$ также имеет место обеднение (т.е. $d(U=0) \neq 0)$, обусловленное диффузией носителей заряда в металл и образованием контактной разности потенциалов $U_{c}[18]$. Величина $U_{c}$ является важной характеристикой полупроводника и, как будет показано, измеряется при помощи рассматриваемого метода диагностики. Представленное на рис. 1 распределение проводимости со скачкообразным изменением $\sigma$ на границе обедненного слоя $x=d$ является приближенным. Более точно распределения концентрации носителей и проводимости в толще полупроводника описываются непрерывными функциями $n(x), \sigma(x)[19]$.

Примеры полученных спектров $Z(f)$ для антенн $1-3$ при $U=0,1.5,5 \mathrm{~V}$ представлены на рис. 2. Спектры $X(f)$ на рис. 2, $b$ соответствуют увеличению толщины обедненного слоя $d$ с ростом напряжения $U$, из-за чего уменьшается емкость контакта (т.е. растет величина реактанса $X)$. Указанное поведение спектров $X(f)$, полученных при отрицательном напряжении смещения 

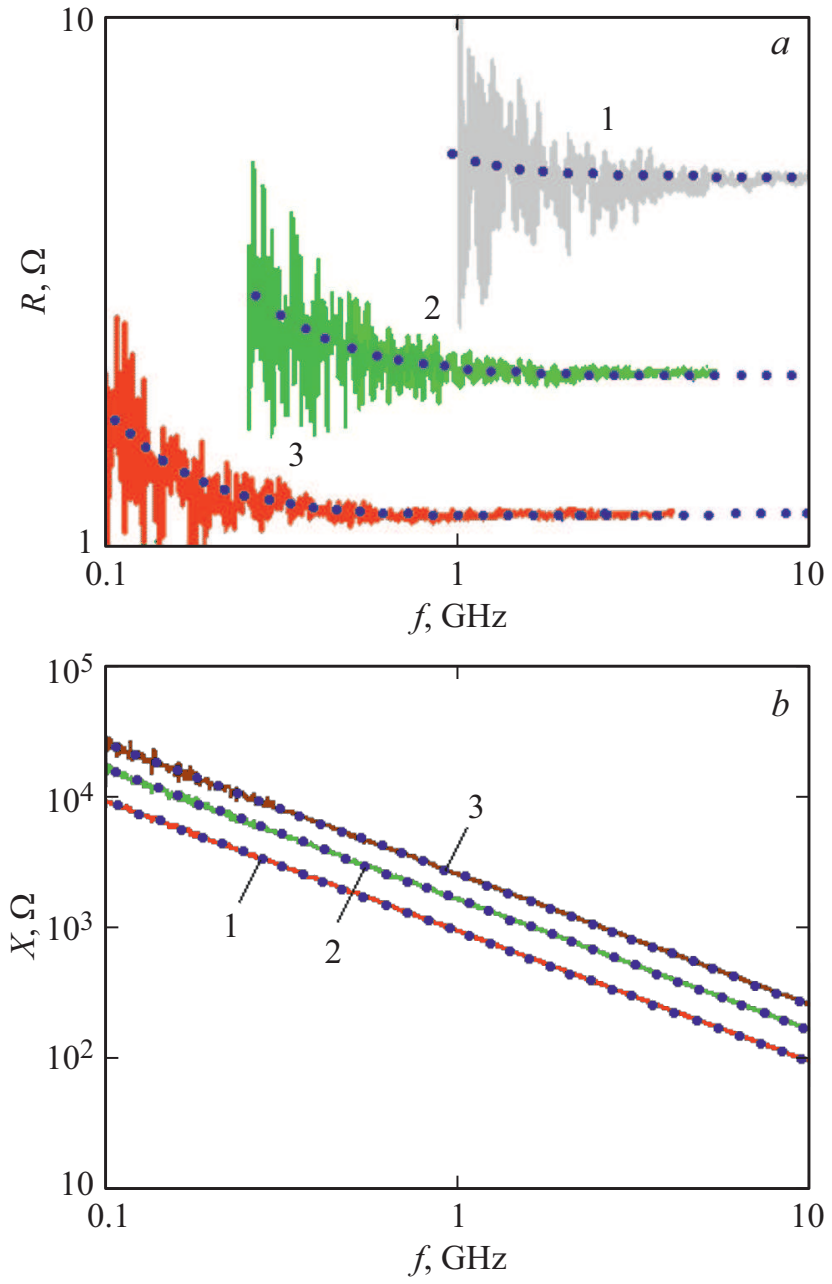

Pис. 2. $a-$ - спектры сопротивления $R$, измеренные антеннами 1-3 при $U=0 . b-$ спектры реактанса $X$, измеренные антенной 1 при $U=0(1), 1.5(2), 5 \mathrm{~V}(3)$. Точки - сглаженные функции $R(f), X(f)$.

на центральном контакте антенны, характерно для полупроводника $n$-типа (в нашем случае $n$-GaAs). C учетом сказанного, приводимые в настоящей работе положительные значения $U$ отвечают отрицательному смещению. Как можно видеть из рис. 2, точность измерения реактанса $X$ оказывается достаточно высокой во всем рассматриваемом частотном диапазоне, в то время как измеренный спектр сопротивления $R(f)$ имеет большой разброс от точки к точке для частот $f<0.5-2 \mathrm{GHz}$ (в зависимости от радиуса антенны $a$ ), из-за чего низкочастотная часть полученных спектров $R(f)$ на рис. 2, a не показана. Очевидно, что точность измерения импеданса наиболее высока при условии $R \sim X \sim Z_{0}$, где $Z_{0}=50 \Omega-$ волновой импеданс СМ зонда. В указанной частотной области имеем $X \gg Z_{0}, R \ll X$, вследствие чего небольшие погрешности изменения коэффициента отражения $\mathrm{S} 11(f)$ приводят к большим скачкам сопротивления $R$. Соответствующая тенденция в поведении функций $R(f)$ видна из рис. 2, $a$ при низких частотах.
Тем не менее большое количество дискретных частот (более 1000), на которых выполнены измерения, позволяет наблюдать рост функций $R(f)$ в низкочастотной части, что, однако, нуждается в дополнительном экспериментальном подтверждении.

\section{2. Метод решения обратной задачи}

Решение обратной задачи в общем виде заключается в определении глубинного профиля проводимости $\sigma(x)$ в полупроводнике из измерений спектра импеданса антенны $Z(f)$. Поиск решения осуществляется с использованием теории, связывающей импеданс коаксиальной антенны с профилем комплексной диэлектрической проницаемости исследуемой среды $\varepsilon(x)=\varepsilon^{\prime}+i \varepsilon^{\prime \prime}(x)$, где в микроволновом диапазоне для полупроводника имеем $\varepsilon^{\prime \prime}(x)=\sigma(x) /\left(2 \pi f \varepsilon_{0}\right), \quad \varepsilon_{0}-$ проницаемость вакуума. Соответствующая аналитическая теория разработана в работе [11] и уже использовалась для решения обратной задачи в [6], а также в компьютерном моделировании [8]. Еще раз подчеркнем, что использование одномерного распределения проводимости $\sigma(x)$ оправдано при условиях $a \ll b, d \ll a$, когда имеет место вертикальный транспорт электронов в обедненном слое [6] и трехмерное растекание тока в невозмущенной области. Важно, что теория [11] исходно была разработана для коаксиального зонда ближнепольного микроскопа. В той ситуации радиусы $a, b$ и высота зонда $h$ (даже при его плотном контакте с поверхностью образца) - это эффективные параметры, для определения которых требовались дополнительные калибровочные измерения на эталонных объектах. В отличие от микроскопа геометрия антенны на рис. 1 точно соответствует модели [11], при этом радиусы $a, b$ и высота $h=0$ являются истинными (не подгоночными) геометрическими характеристиками, для определения которых не требуются дополнительные измерения. Указанное обстоятельство определило выбор конфигурации антенн в настоящей работе.

Принципиальной особенностью рассматриваемой задачи является ее математическая некорректность, заключающаяся в том, что приближенными решениями, с высокой точностью отвечающими экспериментальному спектру $Z(f)$, является бесконечное множество разнообразных функций $\sigma(x)$, весьма далеких от истинного распределения проводимости. Возможность успешного решения подобных обратных задач состоит в том, чтобы выбрать регуляризирующий алгоритм, ограничивающий класс искомых функций с учетом имеющейся априорной информации об искомом точном решении. В используемом нами подходе регуляризацией является предположение, что профиль представляет собой кусочнооднородную функцию, характеризуемую конечным набором неизвестных параметров, подлежащих определению в процессе решения. Простейшей аппроксимацией для $\sigma(x)$ является профиль, показанный на рис. 1, который характеризуется набором из трех параметров, 
а именно вектором $\mathbf{G}=\left\{d, \sigma_{d}, \sigma_{0}\right\}$. В этом случае решение обратной задачи заключается в поиске компонент вектора $\mathbf{G}$ по данным измерений импеданса $Z_{e}\left(f_{k}\right)$ на нескольких частотах $f_{k}(k=1,2, \ldots, N)$ при фиксированном напряжении $U$.

Поиск решения осуществлялся путем минимизации функции невязки:

$$
\begin{aligned}
& F[\mathbf{G}(U)]= \\
& =\sum_{k=1}^{N}\left\{\frac{\left[R_{e}\left(f_{k}, U\right)-R\left(\mathbf{G}, f_{k}\right)\right]^{2}+\left[X_{e}\left(f_{k}, U\right)-X\left(\mathbf{G}, f_{k}\right)\right]^{2}}{R_{e}\left(f_{k}, U\right)^{2}+X_{e}\left(f_{k}, U\right)^{2}}\right\}^{1 / 2},
\end{aligned}
$$

где $Z(\mathbf{G}, f)=R(\mathbf{G}, f)-i X(\mathbf{G}, f)$ - результат расчета импеданса $Z$ для набора компонент вектора $\mathbf{G}$, начиная от выбранного начального вектора $\mathbf{G}_{0}$. В качестве решения обратной задачи принимается вектор $\mathbf{G}=\mathbf{G}_{\min }$, для которого функция (1) достигает минимума. Нами создана компьютерная программа, минимизирующая функцию (1) на основе алгоритма Нелдера-Мида (см. также [8]). Программа осуществляет итерационный спуск от $\mathbf{G}_{0}$ к $\mathbf{G}_{\min }$ с уменьшением значения невязки $F$ на каждом шаге. Пошаговая динамика изменения функции $F$ отображается на экране компьютера в реальном времени. Процедура минимизации автоматически останавливается после того, как функция $F$ перестает меняться от шага к шагу с некоторой заданной точностью. В окне итогового протокола выводятся искомые компоненты вектора $\mathbf{G}$, минимальное значение невязки $F_{\min }$ и число итераций $N_{\text {it. }}$ Время поиска решения зависит от количества искомых параметров образца и от выбора начального вектора $\mathbf{G}_{0}$. Для вышеуказанного набора из трех параметров обычно имеем $N_{\text {it }}=200-300$ при общем времени сходимости 3-5 min. Повторяя процесс решения при нескольких значениях $U$, получим зависимости $d(U), \sigma_{d}(U), \sigma_{0}=$ const.

Следующая задача состоит в определении электрических характеристик полупроводника из полученного решения $\sigma(x, U)$. С этой целью используем соотношение для толщины обедненного слоя $d(U)$, полученное в приближении Шоттки. В этом приближении решается уравнение Пуассона для потенциала $\varphi(x)$ в полупроводнике [18]:

$$
\frac{d^{2} \varphi}{d x^{2}}=\frac{e}{\varepsilon^{\prime} \varepsilon_{0}}\left[n(x)-n_{0}\right]
$$

в предположении, что $n(x) \ll n_{0} \quad$ при $\quad x<d$ (в уравнении (2) $e$ - заряд электрона). При $x>d$ предполагается $n(x)=n_{0}=$ const, т. е. на границе обедненного слоя концентрация меняется скачкообразно. Пренебрегая функцией $n(x)$ в правой части (2) и полагая $\varphi(x=0)=0, \varphi(x \geq d)=U_{c}+U=$ const, имеем

$$
\varphi(x)= \begin{cases}-\frac{e n_{0}}{2 \varepsilon^{\prime} \varepsilon_{0}}(d-x)^{2}+\left(U_{c}+U\right), & x \leq d, \\ U_{c}+U, & x>d,\end{cases}
$$

где

$$
d=d(U)=\sqrt{\frac{2 \varepsilon^{\prime} \varepsilon_{0}\left(U_{c}+U\right)}{e n_{0}}}
$$

есть функция невозмущенной концентрации $n_{0}$. В результате, имея зависимость $d(U)$, найдем по формуле (4) концентрацию $n_{0}$ и барьерную разность потенциалов $U_{c}$. Зная из решения обратной задачи невозмущенную проводимость $\sigma_{0}$, определим подвижность носителей заряда $\mu=\sigma_{0} /\left(e n_{0}\right)$. Таким образом, получаем полный набор искомых электрофизических характеристик полупроводника.

\section{3. Определение электрических характеристик}

Минимизация невязки (1) производилась с использованием экспериментальных данных для $N=11-13$ частот, равномерно заполняющих диапазоны $0.1-10$, $0.05-3,0.02-1 \mathrm{GHz}$ для антенн $1-3$ соответственно. Сопротивление $R_{e}$ и реактанс $X_{e}$ принимались равными соответствующим значениям для сглаженных спектров (точечные кривые в примерах на рис. 2). Напряжение смещения $U$ составляло $0,0.5,1, \ldots, 4.5,5 \mathrm{~V}$. Действительная часть диэлектрической проницаемости GaAs принята равной $\varepsilon^{\prime}=12.9$. В простейшем трехпараметрическом представлении профиля $\sigma(x)$ были получены зависимости $d(U), \sigma_{d}(U), \sigma_{0}$ для каждой из трех использованных антенн. Величина $\sigma_{0}$, естественно, от напряжения $U$ не зависит. В частности, приведенные в таблице средние по всем $U$ значения $\sigma_{0}$ получены при стандартном отклонении 2-5\%. Решениям обратной задачи во всех случаях соответствовали весьма малые значения невязки в минимуме $F=(4-6) \cdot 10^{-5}$. Это означает, что найденные параметры трехпараметрической аппроксимации функции $\sigma(x, U)$ и выполненные для этих параметров расчеты спектров $Z(f, U)$ с высокой точностью отвечают экспериментальным спектрам $Z_{e}(f, U)$.

Зависимость $d(U)$ была использована для определения параметров $U_{c}$ и $n_{0}$. С этой целью формула (4) преобразована к виду

$$
\left[d(U) / d_{0}\right]^{2}=\left(1+U / U_{c}\right)
$$

где $d_{0}=d(U=0)$ в (4). Таким образом, зависимость $y=\left[d(U) / d_{0}\right]^{2}$, как функция нормированного напряжения $u=1+U / U_{c}$, должна представлять собой прямую линию $y=u$, в том случае, если приближение (4) адекватно описывает толщину обедненного слоя. На рис. 3 в качестве примера представлена зависимость (5) для антенны 1 при $U_{c}=0.75 \mathrm{~V}, d_{0}=77.5 \mathrm{~nm}$ вместе с полученными из решения обратной задачи значениями $d(U)$. Параметры $U_{c}$ и $d_{0}$ найдены путем аппроксимации экспериментальной зависимости $d(U)^{2}$ прямой линией методом наименьших квадратов. Можно видеть, что для приведенных значений $U_{c}$ и $d_{0}$ экспериментальные точки 
Электрофизические характеристики полупроводника

\begin{tabular}{c|c|c|c|c|c}
\hline$a, \mu \mathrm{m}$ & $U_{c}, \mathrm{~V}$ & $\sigma_{0},(\Omega \cdot \mathrm{cm})^{-1}$ & $n_{0}, \mathrm{~cm}^{-3}$ & $\mu, \mathrm{cm}^{2} /(\mathrm{V} \cdot \mathrm{s})$ & $10^{5} \sigma_{d}(U=0-5 \mathrm{~V}),(\Omega \cdot \mathrm{cm})^{-1}$ \\
\hline 6 & 0.75 & 86.4 & $1.78 \cdot 10^{17}$ & $3.03 \cdot 10^{3}$ & $0.65-1.8$ \\
13.5 & 0.75 & 81.3 & $1.90 \cdot 10^{17}$ & $2.67 \cdot 10^{3}$ & $0.32-0.88$ \\
28.5 & 0.75 & 82.7 & $2.03 \cdot 10^{17}$ & $2.55 \cdot 10^{3}$ & $0.14-0.21$
\end{tabular}

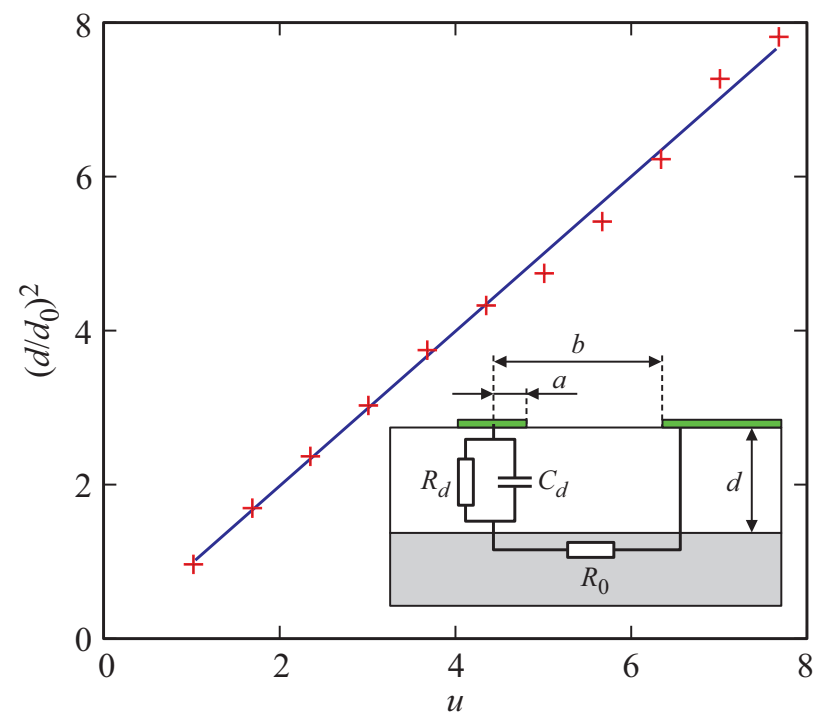

Рис. 3. Квадрат нормированной толщины обедненного слоя, как функция нормированного напряжения. Значки - решение обратной задачи по экспериментальным данным для антенны 1 , сплошная линия - функция $y=u$. На вставке эквивалентная схема образца с обедненным слоем.

очень хорошо ложатся на прямую линию $y=u$ во всем рассматриваемом диапазоне напряжений $U$. Среднеквадратичный разброс экспериментальных точек относительно прямой линии не превышает 3\%. Например, в приведенном на рис. 3 примере из решения обратной задачи имеем $d(U=0)=76.3 \mathrm{~nm}$ (ср. с полученной величиной $d_{0}$ ). Используя найденные значения $d_{0}, U_{c}$, из формулы (4) получим невозмущенную концентрацию $n_{0}=2 \varepsilon^{\prime} \varepsilon_{0} U_{c} /\left(e d_{0}^{2}\right)$ и затем определим подвижность $\mu=\sigma_{0} /\left(e n_{0}\right)$. Электрические характеристики полупроводника для каждой антенны приведены в таблице.

Для сравнения были выполнены холловские четырехзондовые измерения электрических характеристик полупроводника в геометрии Ван-дер-Пау. С этой целью на квадратном фрагменте подложки GaAs, идентичной по параметрам исследуемому образцу, были сформированы металлические контакты. В проведенных измерениях был подтвержден $n$-тип подложки и получены удельная проводимость $\sigma_{0}=117(\Omega \cdot \mathrm{cm})^{-1}$, концентрация электронов $n_{0}=2.00 \cdot 10^{17} \mathrm{~cm}^{-3}$, холловская подвижность $\mu_{\mathrm{H}}=3.65 \cdot 10^{3} \mathrm{~cm}^{2} /(\mathrm{V} \cdot \mathrm{s})$. Можно констатировать удовлетворительное взаимное соответствие полученных данных.
Эффективная проводимость $\sigma_{d}$ обедненного слоя также определена в процессе решения обратной задачи (см. таблицу). Заметим, что для определения невозмущенных параметров $n_{0}, \sigma_{0}, \mu$ полупроводника не требуется находить проводимость $\sigma_{d}$. Однако зависимость $\sigma_{d}(U)$ может представлять интерес для изучения физики процесса обеднения. Приближения (3), (4) не дают величину $\sigma_{d}$, которая характеризует резистивные свойства обедненного слоя. Рассматриваемому трехпараметрическому представлению образца с обедненным слоем отвечает эквивалентная схема, показанная на вставке к рис. 3. В обедненном слое имеет место вертикальный токовый транспорт, т.е. электрическое поле под центральным контактом антенны ортогонально поверхности образца. Магнитное поле вблизи контакта пренебрежимо мало. В такой ситуации емкость и сопротивление обедненного слоя - это характеристики плоского цилиндрического конденсатора с утечкой:

$$
C_{d}=\varepsilon_{0} \varepsilon^{\prime} S_{a} / d, \quad R_{d}=d /\left(\sigma_{d} S_{a}\right),
$$

где $S_{a}=\pi a^{2}$ - площадь центрального контакта антенны. В невозмущенной области полупроводника имеет место трехмерное растекание тока. Геометрия поля и тока здесь центрально симметричная, поэтому электрическая энергия в основном сосредоточена вблизи центрального контакта антенны в области радиуса $a$. Сопротивление растекания может быть вычислено по формуле [20]:

$$
R_{0}=\frac{1}{2 \pi}\left[\sqrt{\frac{\omega \mu_{0}}{2 \sigma_{0}}} \ln \left(\frac{b}{a}\right)+\frac{1}{\sigma_{0} a} \operatorname{tg}^{-1}\left(\frac{b}{a}\right)\right],
$$

где $\omega=2 \pi f, \mu_{0}-$ магнитная проницаемость вакуума. Нетрудно убедиться, что эквивалентная схема на рис. 3, где для расчета сосредоточенных элементов (6), (7) используются полученные из решения обратной задачи параметры $d(U), \sigma_{d}(U), \sigma_{0}$, дает значения импеданса $Z(f, U)$, близкие к результатам измерений (примеры на рис. 2). В рассматриваемом частотном диапазоне в обедненном слое ток смещения преобладает над током проводимости, в результате чего имеем

$$
X \approx \frac{d}{\varepsilon_{0} \varepsilon^{\prime} S_{a} \omega}, \quad R \approx \frac{\sigma_{d} d}{\left(\varepsilon_{d} \varepsilon^{\prime} \omega\right)^{2} S_{a}}+R_{0} .
$$

Видно, что $R_{0}$ есть не зависящее от $f$ сопротивление в высокочастотной области спектров на рис. 2, $a$. Действительно, в рассматриваемом случае первое слагаемое формулы (7) пренебрежимо мало и при $b \gg a$ получаем значения $R_{0} \approx 1 /\left(4 \sigma_{0} a\right)$, близкие к измерениям 
на рис. 2,a. Указанное соотношение слагаемых (7) означает, что электрическое поле антенны проникает в полупроводник на глубину $\sim a \ll \delta_{s k}=\sqrt{2 / \omega \mu_{0} \sigma_{0}}$, где $\delta_{s k}$ - глубина скин-слоя.

Как уже было сказано, трехпараметрическое представление со скачкообразным изменением проводимости на границе обедненного слоя $x=d$ является довольно грубой аппроксимацией непрерывного профиля $\sigma(x)$. В таком приближении проводимость оказывается искусственно распределенной по всей толщине обедненного слоя $d$. В результате параметр $\sigma_{d}$ следует рассматривать, как эффективную проводимость. Предлагаемый подход в принципе дает возможность исследовать более сложные представления функции $\sigma(x)$, которые позволят судить о структуре этой функции. Типичным свойством некорректных обратных задач является бесконечное множество решений, с заданной точностью удовлетворяющих одним и тем же экспериментальным данным, среди которых существует искомая непрерывная функция $\sigma(x)$. Можно надеяться, что описание этой функции при помощи небольшого числа (более 3-х) подлежащих определению параметров даст адекватное (регуляризованное) решение. Соответствующие исследования требуют повышения точности измерения низкочастотной части спектра $R(f)$, информативной к структурным параметрам профиля $\sigma(x)$. Выполненные в настоящей работе измерения (рис. 2, $a$ ) сопровождались аномально большой погрешностью в этой части спектров. Еще раз подчеркнем, что качество решения основной задачи настоящей работы - определения электрических характеристик полупроводника, не зависит от точности представления функции $\sigma(x)$ в обедненном слое.

\section{Заключение}

В работе экспериментально апробирован метод, который позволяет с микронным латеральным разрешением определить электрофизические характеристики полупроводника по данным измерений микроволнового спектра комплексного импеданса коаксиального зонда. Зондовая (антенная) система была сформирована непосредственно на поверхности образца. После выполненных измерений матрица антенн может быть удалена при помощи несложной технологической операции без изменения характеристик полупроводника. Таким образом, метод можно рассматривать, как неразрушающий. Традиционный холловский метод измерения электрофизических параметров полупроводников [21] может иметь латеральное разрешение $\sim 10 \mu \mathrm{m}$ в геометрии крестообразных электрических контактов (метод холловского креста). Данный разрушающий метод применяется к тонким пленкам на полуизолирующей подложке, либо проводящим каналам с двумерным газом носителей заряда (например, дельта-легированные или транзисторные структуры), т.е. метод имеет ограниченную применимость. Разработанный нами подход может применяться к более широкому классу полупроводниковых образцов и особенно актуален, когда требуется обеспечить микронное разрешение. Представляется потенциально возможным проведение аналогичных бесконтактных измерений при помощи сканирующего микроволнового микроскопа, что требует отдельного исследования. Дальнейшее развитие метода может быть связано с исследованием полупроводниковых структур - пленочных, селективно легированных, транзисторных с проводящим каналом и др. Изучение распределения проводимости в обедненном слое полупроводника станет возможно, если удастся повысить точность измерений низкочастотной части спектра сопротивления антенны.

\section{Благодарности}

Авторы признательны С.А. Краеву и Е.А. Архиповой за изготовление антенной решетки на поверхности пластины GaAs, а также C.A. Королеву за выполненные им холловские четырехзондовые измерения электрофизических параметров полупроводника.

\section{Финансирование работы}

Работа поддержана грантом Российского фонда фундаментальных исследований № 18-02-00914 и госзаданием Института физики микроструктур РАН (тема № 00352019-0024). Использовано оборудование Центра коллективного пользования „Физика и технология микро- и наноструктур“.

\section{Конфликт интересов}

Авторы заявляют, что у них нет конфликта интересов.

\section{Список литературы}

[1] Lehovec K. // Appl. Phys. Lett. 1974. Vol. 25. N 5. P. 279-281.

[2] Das M.B., Kim B. // IEEE Tr. Electron. Devices. 1982. Vol. ED-29. N 2. P. 205-211.

[3] Donzelli G.P., Guarni G., Sveto V. // IEEE Tr. Electron. Devices. 1979. Vol. ED-26. N 5. P. 811-815.

[4] Paszkiewicz B., Wosko M., Paszkiewicz R., Tlaczala M. // Phys. Stat. Solidi C. 2013. Vol. 10. N 3. P. 490-493.

[5] Vostokov N.V., Shashkin V.I. // IEEE Tr. Electron. Devices. 2017. Vol. 64. N 1. P. 109-114.

[6] Reznik A.N., Vostokov N.V., Vdovicheva N.K., Korolyov S.A., Shashkin V.I. // J. Appl. Phys. 2017. Vol. 122. P. 244505: 1-9.

[7] Vostokov N.V., Koblov E.A., Korolyov S.A., Revin M.V., Shashkin V.I. // IEEE Tr. Electron. Devices. 2018. Vol. 65. N 4. P. $1327-1332$.

[8] Резник А.Н., Вдовичева Н.К. // ЖТФ. 2019. Т. 89. Вып. 11. C. $1813-1818$.

[9] Imtiaz A., Baldwin T., Nembach H.T., Wallis T.M., Kabos P. // Appl. Phys. Lett. 2007. Vol. 90. P. 23105: 1-3.

[10] Laji K., Kundhikanjana W., Kelly M.A., Shen Z.-X. // Appl. Nanosci. 2011. P. 13-18.

[11] Reznik A.N., Korolyov S.A. // J. Appl. Phys. 2016. Vol. 119. P. 094504: 1-10. 
[12] Korolyov S.A., Reznik A.N. // Rev. Sci. Instrum. 2018. Vol. 89. P. 023706: 1-9.

[13] Buersgens F., Kersting R., Chen H.-T. // Appl. Phys. Lett. 2006. Vol. 88. P. 112115: 1-3.

[14] Трухин В.Н., Голубок А.О., Лютецкий А.В., Матвеев Б.А., Пихтин Н.А., Самойлов Л.Л., Сапожников И.Д., Тарасов И.С., Фельштын М.Л. // Изв. вузов. Радиофизика. 2011. T. 54. № 8-9. C. 640-648.

[15] Huber H.P., Humer I., Hochleitner M., Fenner M. et al. // J. Appl. Phys. 2012. Vol. 111. P. 014301: 1-10.

[16] Amster O., Stanke F., Friedman S., Yang Y., DixonWarren St.J., Drevniok B. // Microelectron. Reliab. 2017. Vol. 76-77. P. 214-217.

[17] Hommel S., Killat N., Altes A., Schveinboeck T., Kreupl F. // Microelectron. Reliab. 2017. Vol. 76-77. P. 218-221.

[18] Бонч-Бруевич В.Л., Калашников С.Г. Физика полупроводников. М.: Мир, 1977. 678 с.

[19] Tsang D.W., Schwarz S.E. // J. Appl. Phys. 1979. Vol. 50. P. 3459-3471.

[20] Dickens L.E. // IEEE Tr. Microwave Theory Tech. 1967. Vol. 15. P. 101-109.

[21] Schroder D.K. Semiconductor material and device characterization. J. Wiley and Sons, Inc., 2006. 779 p. 